Cahiers de recherches médiévales

Journal of medieval studies

Un roi re-né dans la foi de son fils : le personnage d'Avennir des Barlaam et Josaphat au Mystère du Roy Avenir.

Marie-Geneviève Grossel

\title{
OpenEdition
}

Journals

Édition électronique

URL : https://journals.openedition.org/crm/971

DOI : $10.4000 / \mathrm{crm} .971$

ISSN : 1955-2424

Éditeur

Honoré Champion

Édition imprimée

Date de publication : 15 décembre 1997

ISSN : 1272-9752

Référence électronique

Marie-Geneviève Grossel, « Un roi re-né dans la foi de son fils : le personnage d'Avennir des Barlaam et Josaphat au Mystère du Roy Avenir. », Cahiers de recherches médiévales [En ligne], 4 | 1997, mis en ligne le 15 janvier 2007, consulté le 15 décembre 2022. URL : http://journals.openedition.org/crm/971 DOI : https://doi.org/10.4000/crm.971

Ce document a été généré automatiquement le 15 décembre 2022.

Tous droits réservés 


\title{
Un roi re-né dans la foi de son fils : le personnage d'Avennir des Barlaam et Josaphat au Mystère du Roy Avenir.
}

\author{
Marie-Geneviève Grossel
}

1 Pour un homme du Moyen Âge, penserions-nous volontiers, l'image du père devait être simple.Sacralisée par le nom de Père donné à Dieu et infiniment répété dans les prières d'un côté, sanctionnée de l'autre par le commandement "Tes père et mère honoreras", la fonction paternelle est en outre amplement exaltée par la chanson de geste où tant de héros se voient dotés - à rebours- d'une longue ascendance, comme si le texte descendait vers des racines toujours reculées dans la nuit du temps. Et pourtant, si le modèle du père est Dieu, devant le père spirituel, abbé, maître ou saint, doit s'effacer le père selon la chair. La tâche néanmoins n'était pas aisée si l'on en prend pour témoin un roman bien connu de l'histoire sacrée, le Barlaam et Josaphat et sa longue histoire. « Il n'y a peut-être pas de texte édifiant dans toute l'histoire de la spiritualité qui ait " accroché » tant de significations diverses et servi de support à des mystiques aussi différentes par leur origine. Cinq religions au moins ont médité sur ce singulier petit roman $»^{1}$ écrivait R. Nelli, en préface à son édition du Barlaam et Josaphat provençal.

2 Pour nous en tenir à la seule religion chrétienne, on sait l'immense succès que remporta cette histoire exemplaire, puisque, traduite dès l'orée du XIII ${ }^{e}$ siècle dans les langues romanes, elle était encore le thème d'un Mystère écrit par Jean du Prieur, à la demande (et avec l'aide ?) du Roi René d'Anjou ${ }^{2}$. Dans toutes ces versions, si le titre unit traditionnellement le couple spirituel du Maître et de son Disciple, le drame se joue en fait entre trois personnages, car le père de Josaphat, le roi Avennir, a une importance qu'aucun des poètes successifs n'a réduite, et ce jusqu'à l'ultime pièce de théâtre, précisément intitulée «Mystère du Roy Avenir », alors que ce dernier est un païen et, de surcroît, un persécuteur endurci de la religion chrétienne. Les unes après les autres, les générations d'écrivains et leur public semblent avoir rêvé sur la relation spéculaire qui 
unit un fils à son père lorsque le fils, par la grâce du baptême, fait renaître celui qui l'a engendré, tandis que la reduplication du couple originel -Avennir-Josaphat- en Père spirituel et son disciple -Barlaam-Josaphat- évoque implicitement le modèle sous-jacent à toute cette catéchèse, la relation mystérieuse qui fait de Dieu le Père et Dieu le Fils une Personne unique.

Chacun à sa manière, chacun selon les moyens que le genre met à sa disposition, nos textes ont travaillé sur une matière identique. Tous partent d'une prémisse incontestée: le roi Avennir éprouve pour son fils une tendresse indéfectible. Dans les versions les plus anciennes, au XIII ${ }^{\mathrm{e}}$ siècle, Avennir est resté longtemps sans avoir d'enfant et il en éprouvait un vif désespoir, ainsi dans la Mise en prose de la version anonyme:

Mais une seule cose li anuioit et grevoit mout, de çou k'il n'avoit nul oir de son cors

ki tenist apres lui sa terre. $(\$ 1)$

Cette absence d'enfant est implicitement reliée par Chardry à la mécréance du roi qui rime de façon significative avec sa mescheance ${ }^{3}$ d'homme stérile

Joie terrienne out a plenté

Trestut a sa volenté,

Fors tant ke ne sout pas ke Deu fu

Ne n'aveit pas enfant eu (vv. $83 \mathrm{sq})$

Ce désir de paternité qui cause sa tristesse est donc un désir charnel et les auteurs le rejettent avec plus ou moins de tolérance parmi les affections périssables du monde d'ici-bas. Pour l'auteur de la version dite Champenoise, qui est de loin le plus moralisateur de tous nos textes, le désir d'une progéniture n'est qu'une autre des manifestations de l'appétit de richesses qui meut les laïques :

Quar dessierroit estre apelez peres, quar ce ast une chose qui moult plait a riche home (p. 30).

7 Plus indulgent, l'auteur de la Mise en Prose préfère imputer le désir d'un fils non aux « riches hommes " mais aux « hauts hommes " ${ }^{4}$; dès lors le prince à naitre s'inscrit dans une lignée, il est l'héritier du royaume, argument qui n'a rien de négatif, même sous la plume d'un clerc, argument qui reviendra souvent par la suite dans la bouche du roi, lorsque son fils unique choisit de se retirer au désert. Nous sommes dans le domaine de la politique, le Moyen Âge dirait plus volontiers des ordres. Et l'auteur provençal de souligner que la stérilité du roi, outre qu'elle «l'accablait de douleur», n'était pas sans incidence sur son règne puisqu'elle «diminuait son prestige». Le poète évoque, tout aussitôt après, la "glorieuse lignée » des chrétiens ${ }^{5}$ pour mieux faire ressortir que cette absence de fils est un avertissement du Ciel, une sanction, mais, en ce début du récit, Avennir ne saurait la comprendre.

Dans les deux pièces de théâtre, les auteurs ont choisi des voies différentes. Le Miracle Nostre Dame par personnages, œuvre aussi courte que parfaitement sobre, s'ouvre sur la présentation de l'enfant par son père; pour la première fois, on nous évoque sa mère, mais c'est pour la dire morte en couches, et le roi Avennir tend à ses astrologues son enfant orphelin afin que les mages lui dévoilent son destin

De savoir ay le cuer en grant

Quelx il sera.

9 Le Mystère du Roy Avenir cultive une tout autre esthétique : il faut attendre le vers 4414 pour que Josaphat naisse, causant la mort de la reine dont nous avions incidemment appris la grossesse au cours d'une scène où le roi la mignotait fort tendrement ${ }^{6}$. Avennir, heureux et fort occupé, ne souffrait nullement de stérilité et n'éprouvait pas 
de manque affectif. Apprenant coup sur coup la mort de son épouse et la bonne santé du nouveau-né, il passe d'un sentiment extrême à l'autre avec une rapidité digne de Gargantua! Mais l'outrance des sentiments et la violence dans leur expression sont des traits permanents de ce personnage. Le plaisir très réel qu'il a pris jusqu'ici à inventer et faire exécuter des supplices inédits pour les chrétiens explique son coup de rage lorsque les astrologues lui annoncent la conversion future de son fils. Voilà Avennir tout prêt à s'en débarrasser

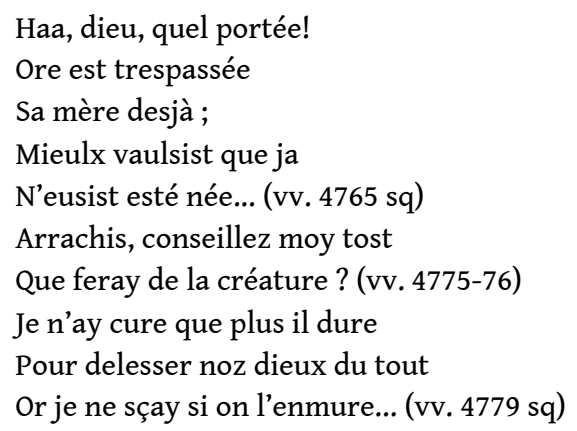

10 Arrachis, qui joue souvent le rôle de «conscience» du roi, se montre heureusement plus pondéré et propose la solution traditionnelle qui est la mise à l'écart du prince.

11 La rapidité propre à ce théâtre, qui fait défiler les scènes sous nos yeux, ne nous permet pas de juger si le conseil d'Arrachis est suivi sur le champ. Dans les versions du XIII ${ }^{e}$ siècle, une seule précise que le magnifique palais où l'on relègue le jeune prince se referme sur lui «quant (le) fils ne fut plus tout à fait un enfant $»^{7}$. Mais il reste assuré que cet exil relatif, loin de refroidir la tendresse du père, ne fait que l'exacerber et ce d'autant plus que Josaphat s'est revélé dès sa naissance un enfant extraordinaire. La Mise en Prose n'omet pas de préciser qu'Avennir venait le plus souvent possible visiter son fils

...ses pères ki le venoit veir cascun jour au matin $u$ au soir. Li enfès vint en l'aage de quinze ans et mout fu grans et biaus mais plus estoit grans ses sens ke ses aages (\& 8)

tandis que les autres versions se contentent d'indiquer que le jeune homme grandit en force, en sagesse, et en beauté. Mais c'est le Mystère qui met l'accent le plus fort sur l'affection paternelle d'Avennir et nous assistons à une sorte de duo lyrique entre le roi et son fils, dans un passage de grande beauté :

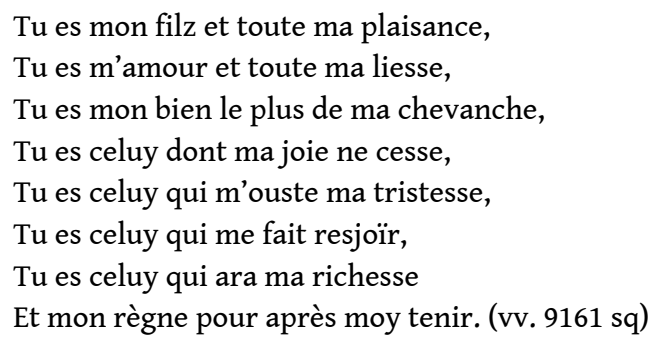

Dans le Mystère, le roi Avennir est un extraverti au cœur plein de passion. Son amour débordant pour Josaphat se double d'une vive admiration qui perce malgré lui lorsque le jeune homme se révèle un redoutable sermonneur.

Nos auteurs ne portent pas de condamnation sur la joie qui saisit un homme quand il devient père d'un fils; si Avennir offre des sacrifices et prodigue les cadeaux pour fêter la naissance d'un successeur, ce qu'on lui reproche, c'est d'offrir son action de grâces à des idoles et non, comme le note l'Anonyme provençal, pour remercier, malheureux 
aveugle qu'il est, le Dieu véritable à qui il devait cette joie ${ }^{8}$. Toute paternité peut donc se lire comme un don de Dieu, encore faut-il savoir reconnaitre cette faveur comme telle et en faire un juste usage. Or quel cadeau plus empoisonné peut-on offrir à un roi farouchement anti-chrétien que de lui apprendre la future sainteté de son fils?

On devine combien cette problématique devait paraître intéressante aux yeux d'un auteur du Moyen Âge. Dans le domaine hagiographique, le plus souvent, une coupure manichéenne sépare les persécuteurs, tout diaboliques, des martyrs, déjà sanctifiés dès cette vie. Lorsqu'un païen en vient à se convertir, de menus indices, parsemés dans le texte, laissent pressentir qu'il s'agissait d'un juste, en intention, sinon en actions Mais il est plus rare qu'un père soit le persécuteur de son enfant. On trouve le cas pour sainte Christine dans la version qu'en a rimée Gautier de Coinci ${ }^{9}$. Cependant tout sentiment de tendresse s'efface vite dans le cœur du père quand il constate la résistance de la fillette - très jeune mais douée d'une force peu commune! Et le poète n'a pas de mot assez dur pour vouer le tyran à la géhenne. Avennir, lui aussi, est un tyran et un sanguinaire, il sera un persécuteur doté d'une longue persévérance et l'on n'imagine guère à le voir agir tout au long du récit (et surtout du Mystère) qu'il puisse se convertir.

Toutes les versions de notre texte s'ouvrent sur la même présentation de ce roi des Indes merveilleuses : on insiste sur sa force, sa noblesse et sa puissance, sa richesse et son intelligence, «le grant sens » dont parle Chardry ${ }^{10}$; seules lui manquent les qualités de l'âme puisqu'il est païen. Et même si l'on sait que suivant la parole «Celui qui n'est pas avec moi est contre moi ", sa haine des Chrétiens atteint un degré qui l'apparente aux pires empereurs des Gesta Martyrum, les Néron, Dèce et autre Dioclétien. On peut se demander pourquoi il éprouve cette horreur du christianisme, lui si noble de cœur. Les poètes se sont naturellement posé cette question qui éclaire, dans la suite, son attitude de père confronté à la foi d'un fils qui l'a renié. Dans la Mise en Prose, Avennir s'est enflammé de haine contre une religion qui lui dérobe les meilleurs de ses courtisans et sujets

Pluisour en i avoit ki avoient esté noble et poissant et mout avant a la court le roi, ki les rikeces et la gloire du monde avoient deguerpi et laissié et estoient devenu moine et ermite pour l'amour Dieu conquerre. (\& 1)

17 Non seulement la conversion des puissants vide la cour mais elle est sans doute également ressentie par le roi comme un désaveu public de sa propre "Weltanschauung", lui qui «as aises et as deslis de cest monde estoit si ententis k'il ne toloit a son cors riens k'il vausist $»^{11}$. La persécution qu'il exerce contre les Chrétiens est une politique soigneusement établie dont il a pesé le pour et le contre; en tant que roi, il veut préserver ses terres et ses sujets d'une croyance qu'il juge funeste et pernicieuse puisqu'elle ôte à ses hommes toute volonté de poursuivre, suivant la tradition ancestrale, leur tâche fixée : avoir des descendants, vivre selon le monde en obéissant à leur seigneur-roi et en faisant fructifier leurs biens; en tant qu'homme, Avennir juge folie de fuir tout ce qui rend agréable l'existence pour s'enfoncer dans une ascèse qui n'est qu'une longue mort anticipée.

On suit particulièrement bien la pensée du roi lorsque, ayant appris la conversion de son fils par Barlaam, il donne l'ordre de chercher le coupable dans tout son royaume. Mais il ne parvient à faire saisir que quelques ermites qui se refusent à trahir leur ami. Le roi éprouve alors comme un besoin de justifier sa conduite devant ces ascètes : 
Vous engigniés ma gent ki joie et desduit lour enseigniés a deguerpir et lour tolés la douçour et le fruit de ceste vie. Et volés en lieu de çou k'il soient povre et caitif et mendiant... Pour çou si ne voel pas ke le simple gent vous croient ne ke nus se praigne a vostre $l i^{12}$. coercition et la torture, lui pour qui les aises du corps ont une telle importance. Il ne daignera pas répondre à l'ermite qui lui rétorque que, pour aimer en vrai roi son peuple, il devrait bien distribuer ses richesses injustement possédées à tant de pauvres qui crient vers lui. Un tel conseil est à ses yeux totalement inconcevable. Mais, dans ce refus de la chair que prône le moine, Avennir ne peut que ranger l'amour qui l'attache à son fils. Déjà lorsqu'il l'allait visiter en son beau palais, l'émerveillement qu'il ressentait à le voir croître ne se doublait-il pas d'une crainte secrète?

Mais mout se doutoit ke il ne le perdesist $u$ par la mort $u$ par ce k'il ne s'aersist a loi crestiene ${ }^{13}$.

Pour Avennir, se convertir, c'est bien mourir, et il ne peut penser que, mourant au monde, son fils lui reste. Bonne par essence, sa paternité est donc devenue condamnable, est condamnée par tout ce qu'elle présente d'exclusive. Sans doute, nos auteurs sont-ils sensibles à des degrés divers, au douloureux presque au tragique qui affecte ici ce personnage. Non seulement il se refuse à perdre son fils pour un Dieu qui lui serait préféré mais le roi se dresse contre la condition humaine même, contre la mort qu'il ne cesse de redouter car elle serait séparation d'avec son fils trop aimé.

Élevé dans un palais merveilleux, au milieu de jardins toujours verts, le prince est entouré de la folâtre compagnie de la jeunesse. Comment Avennir peut-il espérer triompher en cette dérisoire tentative de recréer le monde, vouloir garder ignorées pour Josaphat les dures nécessités de la vie qui passe? C'est dans le cœur même de l'homme, animal triste, que gît le mal d'être et voilà que le prince se languit et tombe malade. À l'apprendre, Avennir éprouve une terrible angoisse. Josaphat, irrité, le presse de questions sur cet emprisonnement immérité et le roi ne peut que répondre qu'il a agi ainsi pour lui procurer la «joie et la liesse $»^{14}$. Mais déjà avant d'avoir quitté cet Eden artificiel pour découvrir l'horreur du vrai monde, déjà l'enfant a perdu tout bonheur de vivre.

Pour convaincu qu'il soit de la folie coupable de son personnage, l'Anonyme de la Mise en prose a su cependant le dessiner d'un crayon léger sans accabler cette paternité luciférienne où le père se voudrait le recréateur du monde autour du fils qu'il a engendré. Quelques traits à peine appuyés donnent au roi Avennir une humanité qu'on ne retrouvera plus ailleurs. Quand les ermites gardent le silence sur la cachette de Barlaam, le roi ordonne de les battre cruellement mais, à ce spectacle, voici que soudain la pitié l'envahit

quant il les vit si plaiés et si navrés, si en ot pitié et dit a cheus ki les batoient

"laissiés les et en aiés merchi. Une autre fois seront tormenté. (\& LXXV) ${ }^{15}$

À une autre reprise, plus subtilement, l'Anonyme nous laisse pressentir que son personnage n'est pas uniquement une brute sanguinaire dotée d'un cœur de père. Avennir, à demi convaincu par son fils et ébranlé par les conversions massives opérées en son royaume, délaisse peu à peu ses cultes ancestraux. Inquiet, le mage Théodas lui propose de regagner Josaphat en le faisant succomber au charme sulfureux de pucelles. On fête la proposition et l'espoir qu'elle soulève par une massive hécatombe. Mais un 
secret dégoût se glisse dans le cœur du roi devant tant de sang et de massacres, préludes à des oraisons plus épurées?

Fu tous li airs corrompus del sanc et de la flairour des boieles des bestes ki ochises i furent $(\& \mathrm{CV})$ de l'Auteur de la version Champenoise: la raison de ses persécutions est directement imputée au diable qui lui a soufflé «le délit du monde » auquel cet hédoniste est fort accroché. Avennir sait trop bien que cette fraille vie n'est que vanité, mais il estime que cette vérité n'est pas bonne à découvrir à Josaphat, encore «enfant» et «novice » en l'existence

Il avoit paour que s'il oïst parler ne des autres chaitivetez de cest siècle qu'il ne l'eüst an despist et qu'il ne meïst son cuer a porchacier la vie pardurable (p. 44)

En vieillissant, on perd assez de cette sotte générosité pour s'attacher aux réalités matérielles. Avennir semble avoir décidé une fois pour toutes de s'en tenir à la vie du corps et aux règles qui organisent son royaume. Fuir au désert lui paraît non seulement un manque à ses devoirs, une ingratitude envers le roi, mais même un acte contre nature, ainsi qu'il le déclare à un ancien de ses proches devenu ermite

n'as mie seulement mise en obli l'amor que j'avoie en toy eins t'as esdresciez contre nature meymes si tu as mis a nonchalor famme et anfanz et paranz et amis (Version champenoise, p. 32).

Fataliste, attaché à son coin de terre, le roi campe un personnage que le doute ne déchire pas et les raisons qu'il invoque devant son fils révolté pour se défendre sonnent étrangement justes à nos oreilles... Ce père, que son fils vient de taxer d'aveugle et de pervers, une fois passée la première colère due à son caractère bouillant, reprend un à un les arguments du jeune chrétien. Certes, il donne une apparence d'hédonisme

Se tu me veïsses trop abandoner es deliz ne es richesces terrienes, je ne m'an merveillasse mie se tu tenisses ma vie a malvaise (ibidem, p. 103)

Mais ce roi sait dans les batailles et le gouvernement "pour le coumun pourfit» endurer le froid et la faim et la soif ; la vertu de largesse ne lui fait pas non plus défaut; et que Josaphat ne lui croie pas un esprit obtus, fermé à toute recherche : jadis, il s'est enquis des autres religions, il a tout écouté et médité ; maintenant il pense être parvenu à la connaissance de la vérité ; elle est celle transmise par les ancêtres, la vie a été donnée par les dieux et la paternité aussi ; il est donc normal, naturel et juste qu'au nom de cette vérité, son fils lui manifeste le respect dû, le suive au lieu de se rebeller et ne porte pas sur son père de jugement, en outre erroné. Se fiant à l'expérience paternelle, Josaphat lui succèdera sans murmure dans ses croyances et à la tête de son royaume.

29 C'est pourquoi notre roi se montre parfaitement logique avec lui-même lorsqu'il unit en une seule réalité l'attachement au monde et l'affection du fils pour le père

Biaus filz, por quoi es tu deceüz por les enchantemanz di un malvais anchantaor que tu as dégerpie l'amor de moi et de monde? (p. 99) 
la Version champenoise se refuse à nous laisser tenter de comprendre Avennir, l'auteur se hâte de préciser que toutes ses paroles (raisonnables ?) ne sont qu'un tissu de mensonges, qu'il n'y croit pas lui-même, comme le juge le perspicace Josaphat

Josaphat pensoit en son cuer a la fauseté des paroles son père. (p. 103)

À aucun moment la croyance et la conduite d'Avennir ne trouvent grâce aux yeux de l'auteur de la Version Champenoise; il a beau nous montrer son personnage partagé entre la colère et son impuissance à faire du mal à un fils chéri ; le conseiller Arrachis a beau lui proposer de céder une moitié du royaume à son héritier pour l'éloigner, puisque Josaphat visiblement convoite le martyre et qu'ainsi, du moins, le père ne le "perdra pas del tout »; et nous de croire sincère l'empressement d'Avennir à accepter! L'auteur prévient tout mouvement trop naïf de son public

Son père ne li fasoit que por lu decevoir por les richesses terriennes (p. 131)

L'anonyme laisse ici percer un peu de son identité, comme nous pouvions la deviner déjà lorsqu'il indiquait en passant que la persécution s'était tout particulièrement attachée aux moines, que, d'autre part, Barlaam était un moine (partout ailleurs, c'est simplement un solitaire ). La probable appartenance de l'auteur au monde monastique en fait un ennemi déclaré de la famille et de ses attaches terrestres. La parabole des trois amis le souligne à l'envi: comment ne pas reconnaître Avennir et son idéal derrière ce père qui amasse des trésors pour ses enfants ; et quel profit en tirera-t-il ?

Il s'est livrez a pardurauble tormant por essanbler l'avoir por cui sui enfent meisme le heent, que nos veons sovant que le filz desssire la mort son père por sa terre tenir et se ses peres n'eüst point de terre, il fust de sa mort dolanz au moins par naturel pitié (p. 73).

L'amour paternel est à rejeter car il est inscrit dans le temps, il faut le soumettre au memento mori: son évanescence lui dénie toute valeur. C'est sans doute la raison pour laquelle Chardry, si économe de moyens par ailleurs, ne manque que deux fois à son laconisme; la première, c'est pour décrire, avec une délectation quasi baroque, le vieillard que rencontre Josaphat en quittant pour la première fois sa prison enchantée, le jeune homme reste stupéfait devant cette horreur ambulante; la seconde, la plus longue, c'est la charmante description du paradis apparue en songe à Josaphat, alors qu'il allait succomber aux charmes d'une entreprenante demoiselle ${ }^{16}$. À l'éphémère s'oppose l'éternité, aux liens charnels du cœur, la foi en l'au-delà.

La très grande brièveté du Miracle par personnages empêche l'auteur de s'attarder longuement sur les causes de la haine du roi Avennir pour les Chrétiens. On apprend la persécution lorsque l'astrologue prédit au roi que son fils fera partie de ceux que «maintenant sur toute riens héz et parfuis ». Mais l'auteur a eu l'idée intéressante de faire de Barlaam l'ancien maître d'hôtel du roi ; il le confond donc avec l'ermite que, dans les autres versions, le roi faisait interroger car il était son ancien familier. Avennir s'irrite de voir son compagnon en loques, comme un fou, un sot que les enfants tournent en dérision et les réponses provocantes du néophyte ne font qu'enfler sa colère contre une secte à ses yeux déjà dangereuse. Mais le dramaturge ne lie pas l'amour paternel et la vie dans le monde ; il préfère faire de son roi un personnage déjà âgé, comme Avennir le rappelle lui-même à son fils

Biau filz, qu'est ce

Pourquoy m'as mis en tel tristesce

Que ma viellesce deshoneures? (vv. 979 sq) 
ès lors et en ce sens, sa conversion finale est facilitée par la force qu'acquiert sur lui le Memento mori. La perspective du Jugement, dont l'évidence aveugle, terrorise et convainc le mage Théodas, entraîne chez Avennir un retour sur lui-même qui fait coïncider un avenir heureux de père et la réconciliation avec le Ciel

Puis que le me loez, je vueil

Biau filz, crestiens devenir,

$\mathrm{Ne}$ jamais je ne vueil tenir

Ce royaume mais le vous quitte.

Et si m'en iray estre hermitte

Pour pardon avoir des meffaiz

Que j'ay ça en arrière faiz (vv. 1661 sq)

Le Mystère a délaissé cette perspective. La conduite d'Avennir dans cette dernière pièce de théâtre reste absolument inexpliquée. Non seulement on ne voit aucun rapport entre les persécutions contre les Chrétiens et les sentiments familiaux du roi, mais ce dernier n'explique jamais sa politique autrement que par l'affirmation répétée de sa croyance en ses dieux. Totalement irrationnelle, sa haine envers les bigots n'en est que plus féroce. En fait, Avennir n'agit jamais de son propre chef $^{17}$ : tout comme sa volonté première de se débarrasser d'un enfant encombré d'un horoscope aussi inquiétant est tout de suite balayée par le conseil d'Arrachis, ses crises de violence contre les Chrétiens coïncident avec les passages sur scène des Diables et Démons divers, qui, pour cocasses qu'ils nous paraissent, n'en représentent pas moins le pouvoir occulte qui agit, telles des marionnettes, Avennir et ses courtisans. Lucifer lui-même donne au roi la dénomination qui le caractérise puisqu'il ne l'appelle jamais que le «pervers roi Avennir ».

Ainsi ce qui était peut-être une maladresse psychologique devient un tout puissant ressort dramatique ; entre la haine aveugle et forcenée qu'il éprouve contre la bigotaille et la non moins violente passion que son fils suscite en lui, Avennir nous paraît un être totalement "schizoïde ${ }^{18}$ que les forces mauvaises agitent en tout sens. Seule la foi de Josaphat peut sauver et sa vie et celle des autres chrétiens. On ne saurait douter de l'issue du combat puisque Dieu le Père intervient plusieurs fois dans la pièce, y dépêchant Gabriel, son messager. Mais on est beaucoup moins assuré de ce qu'il adviendra d'Avennir, personnage décidémént noir et dont l'intérêt semble bien davantage d'appartenir au monde démoniaque que d'être le père d'un saint... Néanmoins, dans le contexte du Mystère, seule la sainteté du Fils peut laisser espérer le salut du Père.

À l'amour d'Avennir pour son fils répond l'amour de Josaphat pour son père ; affirmée avec plus ou moins de force suivant les auteurs, cette affection reste présente dans toute l'histoire de notre récit. Le Miracle Nostre Dame par personnages est celui qui rapproche le plus les deux hommes par la bouche du messager, revenu avec des nouvelles du palais isolé

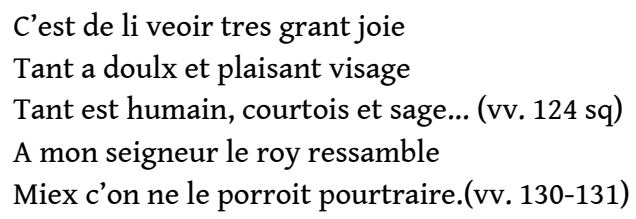

Mais cette ressemblance n'est qu'apparente, ou plutôt l'être profond d'Avennir ne correspond pas à sa courtoise écorce. 

ces lois morales si souvent rappelées - que le christianisme ne saurait récuser. Pas un de nos auteurs n'omet ce petit détail hautement symbolique: lors de la controverse organisée par Avennir entre païens et chrétiens, le roi invite son fils à prendre place auprès de lui ; mais la déférence et le respect poussent le jeune prince à préférer s'asseoir au pied du trône de son père. Ce respect est tout particulièrement accentué dans le Mystère, lorsqu'Avennir rend sa première visite à Josaphat. Le fils lui donne du Monseigneur et il ose à peine accepter de jouer une partie d'échecs contre son père car il ne peut même s'imaginer qu'il le ferait mat

N'esse pas pour moy vitupere

De telle follie entreprendre

Que de juer contre mon père?

41 Pourtant ce fils si courtoisement soumis s'avère bientôt aussi indomptable que le roi. Aux menaces et insultes, il ne répond pas sur le même ton, mais ses propos sont aussi définitifs

Vit-on jamais père se courroucer d'un gain réalisé par son fils? faut-il compter son père parmi ses ennemis ? Je te déclare que je ne t'appellerai plus mon père, que je te fuirai comme l'homme fuit le serpent tant que je te verrai dans ce mauvais vouloir ${ }^{19}$.

Le «Nom du Père», en effet, crée des obligations réciproques et si le fils rebelle est haïssable, le père bourreau est une abomination de la nature.

Si tu veux livrer ton fils à la mort et aux supplices, si tu veux le faire crucifier, tu feras là une chose odieuse et contre nature. Tu ne pourras plus être appelé père ${ }^{20}$.

Avennir ressent avec une douloureuse stupeur la béance qui a fait de son fils, cet autre lui-même, un étranger

Or suis roy et mon fils hermite!

Or me faiz en paine et en doleur

Apeler ma vie maudicte! ${ }^{21}$

Si Avennir assure qu'une telle douleur le fera très vite mourir, Josaphat lui renvoie avec usure l'argument et opère sur son père le même chantage affectif: qu'il le laisse rejoindre au désert Barlaam, ou bien il mourra de désespoir en son royaume. Chantage d'autant plus redoutable que nous avons déjà vu le prince s'aliter et languir, perdant tout goût de vivre lorsque sa prison dorée était devenue pour lui un reclusoir dénué de sens. C'est précisément à ce moment-là que Josaphat avait cherché à percer le secret qui entourait son existence. Cependant il restait alors assuré que son père agissait de cette énigmatique façon dans l'intérêt de son fils «pour son preu ». Une fois opérées les fatidiques rencontres avec la vieillesse, la maladie et la mort, l'enfant s'applique à dissimuler au roi le trouble qui s'est emparé de son âme. Aveugles, les "yeux du cœur " fermés, Avennir veut que son fils partage sa cécité : n'ira-t-il pas jusqu'à faire ôter des chemins où s'avance son Unique toute « ordure » qui pourrait les souiller? Les yeux de Josaphat désormais se sont ouverts ; plus le jeune homme s'avance vers la lumière, plus son père s'enfonce dans les ténèbres, tant la souffrance de perdre Josaphat lui fait «le cœur noir».

Prévenu providentiellement, en effet, Barlaam est arrivé auprès du jeune prince qui désespère d'avoir " perdu le trésor » du savoir puisque son père a exilé tous les ermites qui, il le pressent, seraient susceptibles de répondre à la question existentielle qui le crucifie. 
Amère est cette vie et pleine de douleur et détresse. Quand je serai mort, qui se souviendra encore de moi ? Y aurait-il un autre monde et une autre vie ?22

\section{formes proches}

Que dirais-tu si tu procurais le salut de ton père? Ne serais-tu pas émerveillé

d'engendrer celui qui t’a engendré ? (version provençale, p. 1121)

Se cuit bien ancores serai il par toi saus et que tu seras peres ton pere (Version

Champenoise p.76)

puisque, à plusieurs reprises par la suite, l'auteur nous peint son héros occupé à prier sans relâche pour un père dont il ne supporte pas de le savoir perdu.

le Miracle Nostre Dame, le père spirituel de Josaphat, Barlaam, en vient à occuper une place prépondérante dans le cœur du Prince. Après la conversion, Josaphat lui donne toujours le nom de "père »: Barlaam n'a-t-il pas pris bien soin de lui enseigner que la naissance coïncide avec l'entrée dans la foi ? Il déclare avoir quarante-cinq ans et, devant l'étonnement du jeune homme qui lui en donnait soixante-dix, il confirme qu'il est bien apparu sur cette terre soixante-dix ans plus tôt mais que seules comptent les années où il avait ouvert les yeux à la vraie lumière. Tout autre temps, comme celui où s'inscrivent l'avant et l'après d'Avennir le païen, n'est rien que la mort. Convaincu, Josaphat décide de tout quitter pour suivre Barlaam

Sachiez qu'avec vous m'en iray

Pere et honeurs, tout laisseray

Pour Dieu servir.(vv. 789 sq)

Barlaam s'y refuse car cette fuite au désert de l'héritier déclencherait une vague de persécutions épouvantables; il n'accepte pas encore pour sa part d'appeler «fils » son 
catéchumène, comme il le fera plus tard dans les solitudes érémitiques. Dans la Mise en prose, il va même jusqu'à fixer au nouveau converti un terme avant qu'ils ne se revoient

Quant tu aras chi esté tant ke tu aras ton père et ses home tourné a nostre foi, si

t'en porras venir a moi.(\& LII).

Nous retrouvons les Ordres du Moyen Âge dans cette volonté de faire remplir à chacun la tâche que Dieu lui a fixée, à sa place, celle de Josaphat étant à la tête du royaume ; l'Anonyme se refuse à sanctionner de son aval le reniement du père de chair par le fils rebelle, tout comme l'ascète se refuse à accepter la vénération du néophyte déjà prêt à le suivre au plus profond désert.

Dans le Miracle par personnages, Barlaam ne se montre pas si exigeant; il conseille simplement la patience et la prière et le jeune homme s'incline mais, lorsque le roi fait courir faussement la nouvelle que Barlaam a été capturé et emprisonné, le désespoir de Josaphat éclate et il affirme alors de façon explicite qu'il tient Barlaam pour son père

Bien m'est source douleur amère

Quant prison est Barlaam mon père,

Mon père voire vraiement

Quant est de m'ame au sauvement (vv. $1045 \mathrm{sq}$ ).

Mais là encore, c'est le Mystère qui se révèle le plus affectif. La séparation de Barlaam d'avec Josaphat son «bel enfant » arrache des larmes au maitre comme au disciple. Et Josaphat, pour retenir son père spirituel, use des termes les plus tendres, les plus chaleureux, les plus pathétiques

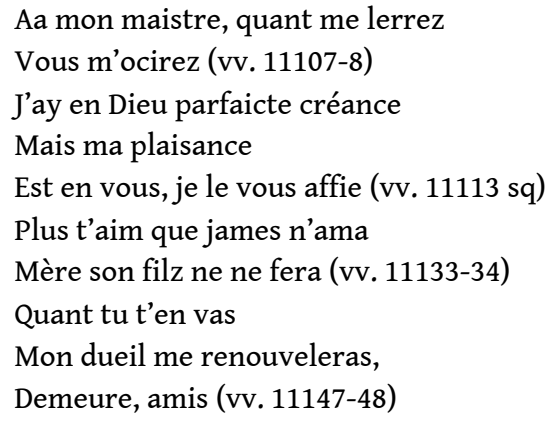

Barlaam a cependant un rôle plus ambigu que celui d'Avennir. Le jeune Josaphat éprouve à son égard un amour plus que filial ; on pense aux chansons de croisade où la jeune fille abandonnée serre contre sa chair la chemise du pélerin lorsqu'on voit le néophyte demander à son maître sur le départ sa tunique d'ascète toute rapiécée, déchirée et trop courte pour la porter secrètement nuit et jour, à même la peau, sous ses habits princiers.

\section{Dans la Version Champenoise, l'exclamation}

Hay! bons mestres, ceste lasse d'arme, ou troverai ele mais si bon pastre con tu li as esté ? (p. 91)

semble mettre le pieux ermite presque au rang de Dieu, le Bon Pasteur, et après son départ la «brebis» pleure de se retrouver "povre orpheline». Et, toujours dans La Version Champenoise, les "compagnons d'éternité ${ }^{27}$ partageront au ciel un bonheur identique et seront couchés côte à côte dans le même tombeau

Il estoit bien droiz que li cors de çaux fusent ensemble en terre don les armes sont ensenble en ciel et qui longuemant avoient esté conpaignon ensenble a lor vie. (p. 157) 
60 À lire ces propos, on pourrait croire que la paternité spirituelle efface la paternité selon le monde; qu'il ne s'agisse d'ailleurs plus de paternité mais d'un nouveau sentiment bien plus fort, c'est ce que suggère la Version Champenoise

Ore ast Josaphat a aise ; or ai quamque il voilt et aime, car quant l'en conmance a amer por amor de Deu, nule naturel amor n'est si granz. (p. 148).

61 La version provençale est plus prudente en opposant l'amour de Dieu à l'amour des hommes, fussent-ils prochains, comme s'opposent transcendance et immanence

Il partit le cœur joyeux comme un enfant qui a depuis longtemps le désir de revoir son père ; mais l'amour de Dieu, s'il se loge dans l'homme est encore plus fervent et plus puissant que l'amour naturel. (p. 1215)

62 Par contre, le Miracle Nostre Dame se contente de juxtaposer les deux affections et, une fois opérée la rapide conversion d'Avennir, père et fils s'en vont ensemble avec tous les courtisans, c'est alors pour le jeune Prince l'occasion d'unir dans la même foi et la même tendresse ses deux pères

Barlaam, père et ami chier...(v. 1701)

.... Diex fait nous a

Grace apperte et miracle gent :

Mon seigneur mon père et sa gent

Ay avec moy si amenez

Pour estre touz crestiennez.(vv. 1706 sq)

63 Il a été établi que la mise en drame du Miracle Nostre Dame par personnages avait pris pour texte de base celui de la Mise en Prose de la version anonyme. Or si l'affection que Josaphat porte à son maître Barlaam, sorte d'adoration, de vénération et de tendresse passionnée, ne cesse de croître et de se voir proclamée, on nous rappelle avec insistance, tout particulièrement dans la Mise en Prose, que jamais Josaphat n'oublie son père naturel, devenu pourtant l'ennemi déclaré de tout ce qu'aime le jeune converti ; ce dernier accepte ici sans murmure la remise de la moitié du royaume et il y accomplit avec justice et exactitude son devoir royal (on ne voit même pas qu'il ait fait pratiquer le baptême en masse de ses sujets! $\left.{ }^{28}\right)$. C'est comme si finalement l'obéissance due le poussait à respecter contre son cœur, contre son aspiration au désert, le choix d'Avennir. Le premier devoir de tout fils semble bien en dernière analyse cette acceptation révérencieuse des droits de la paternité, aussi bien paternité charnelle que spirituelle car Josaphat a laissé partir Barlaam sans le suivre; les deux paternités viennent alors s'unir pour former l'image de la Paternité véritable, référence divine, tandis que Josaphat, qui obéit sans discuter, s'attache même à aimer la tâche imposée, devenant par delà les oppositions et les traverses le Fils véritable. Car Josaphat, une fois roi, se révèle le parfait monarque, il suit les lois de Dieu et se montre pour ses sujets un autre père

Il était le père et la providence des orphelins, des pauvres et des malheureux ${ }^{29}$.

Il donne donc de lui l'image christique du souverain des pauvres. À la fois parce qu'il entend raconter ces merveilles, et parce que l'absence de son fils lui est insupportable, Avennir peu à peu rentre en lui-même. Dans les romans, cette conversion a été annoncée de loin, on l'avait déjà pressentie lorsque le mage Théodas avait présenté au roi l'amour sensuel comme ultime recours contre la croyance des chrétiens. Dans le Mystère, par contre, la conversion d'Avennir sonne comme une grâce inattendue et 
gratuite. Nous voyons bien le roi déchiré de douleur lorsque Josaphat s'en va pour recevoir sa part d'héritage

Mon fils s'en va... (v. 14019)

Je n'atens sulement que l'eure

Que mort sourviengne

A mon corps et qu'ele me tiengne

Pour moy occire.

Quant voy et si fault qu'il avaingne

Mon filz partir,

De plourer ne me puis tenir (vv. $14027 \mathrm{sq}$ )

Mais leur cohabitation est devenue impossible comme leurs rencontres et Avennir, impénitent, confie tant de chagrin à la grâce de Jupiter... Ce n'est que plus tard qu'il demande à son maitre d'hôtel de plus amples renseignements sur une religion qu'il a passionnément haïe et pourchassée, mais qui lui a pris définitivement son fils tant aimé. Il semble alors que pour franchir la distance qui désormais le sépare, dans l'espace et dans les sentiments, de Josaphat, il accepte la suprême humiliation: reconnaître qu'on s'est trompé du tout, renier tout ce que l'on a été... et estimer juste la terrible réparation.

Mais quelle peine éprouverait-il à ce faire quand la conversion suscite chez le père comme le fils une joie si parfaite

Li rois ne se pot saouler de baisier son fill $\mathrm{s}^{30}$.

Il n'y a guère que le très sceptique moine de la Version Champenoise pour émettre quelques doutes sur le pardon miséricordieux accordé par Dieu à son inlassable persécuteur. Toutes les autres versions éclatent de l'allégresse du père et du fils enfin réunis. Voilà Josaphat qui se fait parrain de son père, qui le baptise Josaphat qui ses filz estoit charnex le leva de fonz et fu ses peres esperite ${ }^{31}$.

Les ténèbres qui l'aveuglaient se sont dissipées, Avennir reconnaît s'être trompé : mais son fils fut pour lui la lumière envoyée par Dieu; il lui demande humblement ce qu'il doit faire; et ainsi il abdique son savoir et sa puissance pour se faire aussi obéissant qu'un enfant devant celui qui le dépasse en sagesse. Le salut d'Avennir vient donc d'avoir su précisément comprendre que la paternité de chair, inscrite dans le temps et périssable, doit s'abolir pour que la relation renaisse en se spiritualisant

«Cher fils, tu n'es pas mon fils mais celui du Père céleste. Quelles grâces ne lui dois-

je pas rendre pour toi! Ennemi de Dieu, j'étais perdu .Maintenant je suis réconcilié avec lui. » Et il baisait son fils ${ }^{32}$.

70 À la leçon première qui ouvrait le récit, toute paternité est don de Dieu, le pénible cheminement d'Avennir ajoute cette conclusion, elle est de Dieu si elle se renonce à elle-même, si l'amour éprouvé pour le fils consiste à le laisser marcher son chemin où, nécessairement, il vous dépassera.

71 Dans cet épurement où Avennir se dépasse lui-même comme homme fini, il accède à la sainteté que lui montrait son fils, il retrouve la leçon que Barlaam en quittant Josaphat lui avait donné à méditer. Et Chardry ajoute de son chef ce commentaire charmant et profond

Car Deu ama tant l'enfant

Kel pere ne vout estre perdant (vv. 2415-2416) 
72 C'est aussi chez Chardry que la douleur de Josaphat à la mort de son père se fait la plus véhémente

Mut l'eimat il estrangement

N'est pas merveilles s'il fu dolent.(vv. 2511-12)

73 Dans les autres textes, nous voyons Josaphat n'oublier son profond chagrin qu'à la perspective d'aller au désert retrouver Barlaam, son père spirituel. À son arrivée, l'ermite ne le reconnaît pas tout de suite car de longs mois de recherche et de macérations ont fané le beau prince, en tout semblable désormais au vieil ascète... Mais celui-ci sait marquer la nécessaire distance, lui qui salue le jeune homme d'un

«Sois le bienvenu, fils de Dieu, héritier du royaume céleste »"33

Avennir aurait-il été pardonné de la violence de son attachement terrestre par sa mort édifiante et son renoncement final à l'amour où il a tout regagné ? Il le semble bien et davantage encore, si nous considérons le récit exemplaire qui vient clore nos textes.

Barlaam s'est endormi pour toujours entre les bras de Josaphat qui, épuisé de larmes et de douleur, tombe de sommeil auprès du corps de son maître à penser et père spirituel. Or un rêve le visite: il est arrivé au Paradis et un ange vient lui remettre deux couronnes; et Josaphat de s'enquérir pour qui sont ces deux couronnes si lumineuses. L'ange lui explique que la première est pour lui qui a sauvé tant d'âmes ; la seconde est aussi pour lui afin qu'il la donne à son père qui s'est réconcilié avec Dieu et a fait pénitence grâce à son fils. Or voici que notre Josaphat en conçoit quelque aigreur car il trouve que son père n'a pas autant de mérite que lui... Il faut que Barlaam lui apparaisse, en vrai maître qu'il est, pour lui faire honte et lui dévoiler que dans la parabole de l'Enfant Prodigue, il tenait soudain le rôle du Frère aîné, jaloux des faveurs du pardon tout puissant.

76 Ainsi Avennir, dont le salut fut si longtemps incertain, devient-il, le temps du rêve, l'objet privilégié de la tendresse du Père Céleste, modèle de tous les pères, non moins modèle pour les fils. Certes, c'est par Josaphat qu'Avennir a obtenu le salut mais la réciproque n'est pas moins vraie et le rôle véritable de Josaphat sur la terre était peutêtre bien de sauver Avennir, roi symbole du royaume... En tout cas, il lui faut apprendre à passer par dessus tout amour propre, comme son père a su le faire, lui qui avait oublié tout ce qui avait été son être pour retrouver son fils perdu, pardonnant envers et contre tout au rebelle qui lui citait, dans les Écritures, ce qui ne pouvait guère emporter son adhésion

«Je suis venu séparer le père du fils et la fille de la mère... Il est écrit : il y a un

temps pour aimer et un temps pour haïr... $»^{34}$

Récurrente dans le récit, la parabole de l'Enfant Prodigue est une des clefs de la sénéfiance du Barlaam et Josaphat, car l'importance accordée au thème de la paternité, venue peut-être de la légende originelle du Bouddha ${ }^{35}$, trouve ici sa justification chrétienne et médiévale. S'il est indéniable que l'amour spirituel dû au Père divin l'emporte infiniment sur tout amour charnel, l'affection du père pour son fils perd tout aspect répréhensible quand, par le renoncement, l'offrande de la chair de sa chair fait de l'enfant «l'hoir du célestial règne».

Ce petit exemplum inattendu qui vient clore le récit, relativisant la sainteté jusque là infaillible de Josaphat, ne donne-t-il pas soudain tout son sens au choix que fit Jehan le Prieur lorsqu'il transforma en Mystère du Roy Avenir le titre traditionnel du Barlaam et Josaphat? 
Aussi charnelle soit-elle, la tendresse paternelle quand elle est sincère et profonde ne peut être un sentiment pervers; elle peut même métamorphoser le "pervers roi Avenir ", cet enfant du diable. Car en elle Dieu se mire et se reconnait ; si loin que soit la brebis égarée, pour peu qu'il trouve en elle cette étincelle du Bien, Dieu ira la chercher par un effet spécial de sa Grâce. Dans cette trame étrange où les personnages sont les reflets les uns des autres, Barlaam le maître spirituel et le père de Josaphat, lui-même père spirituel de son géniteur, on peut discerner une sorte de fascination pour le lien qui unit un homme à son fils, peut-être au Moyen Âge de tous les liens d'ici-bas le seul qui ne puisse jamais être absolument méprisé?

\section{NOTES}

1. R. Nelli, Les troubadours, Jaufré, Flamenca, Barlaam et Josaphat, Bibliothèque Européenne, Desclée de Brouwer, tome 1, 1960, p.1068.

2. La présente étude, outre le Barlaam et Josaphat «provençal » de la fin du XIII ${ }^{-}-$début du $\mathrm{XIV}^{\mathrm{e}}$ siècles (édition citée note ci-dessus) s'appuie sur J. Sonet, Le roman de Barlaam et Josaphat (tome II, la mise en prose de la version anonyme française), Namur-Paris, 1952 ; Chardry's Josaphaz, Set Dormanz und Petit Plet, herausgegeben von J. Koch, Heilbronn, 1879 ; L'histoire de Barlaam et Josaphat, version champenoise, établie par L. Mills, Genève, Droz, 1973 ; Les Miracles-Nostre-Dame par personnages, tome III, édités par G. Paris et U. Robert, Paris, SATF, 1878 ; Le Mystère du Roy Avenir de Jean du Prier, édité par A. Meiller, Genève, TLF, 1970. Cette liste respecte la chronologie telle qu'ont pu l'établir les spécialistes de cette œuvre (notamment J. Sonet). Nous n'avons pu ajouter à notre tableau l'œuvre de Gui de Cambrai (éditée par Armstrong et écrite entre la Mise en Prose de la version anonyme et La version champenoise (vv. 1220-1225), un des ouvrages devenus très difficilement accessibles par les nouvelles règles du Prêt-Inter-Bibliothèques.

3. Chardry, op. cité, rimes des vv. 91-92.

4. Mise en prose de la version anonyme, op. cité, \& I.

5. op. cité, p. 1073.

6. On nous l'apprend au vers 1722 du Mystère. «Elle est plaine jusques aux yeux » dit le courtisan, les vers où la reine le confirme au roi sont les vv. 2816-2822.

7. Barlaam et Josaphat provençal, p. 1083, les autres versions laissent entendre que Josaphat fut relégué dès la prédiction en son reclusoir artificiel, dans le Mystère, on a même l'impression que le père ne visite que rarement son fils, qu'il découvre à son adolescence, mais c'est un « coup de foudre »!

8.op. cité.p.1081.

9. Gautier de Coincy's Christinenleben, éditée par C. Ott, Erlangen, 1922.

10. au v. 80.

11. Ibid.

12. Mise en Prose de la version anonyme, \& LXVII.

13. Ibid., \& VIII.

14. Version champenoise, p. 45. 
15. Il les fera cependant supplicier devant leur volonté affichée de martyre. Mais on ne peut comparer cela avec le Mystère où même le compagnon du roi devenu ermite, qui a dans toutes nos versions la vie sauve par suite d'une promesse anticipée arrachée au roi au début de la conversation, est dûment repris dans la suite de la pièce pour subir, comme tous les autres, un supplice complaisamment détaillé!

16. Description du vieillard, vv. 622-634 et description du paradis, vv. 1958-2028.

17. Personnage d'une violence extrême, Avennir est en réalité la proie de ses passions. Le dramaturge incarne tous ces mouvements de l'âme quand il introduit ses diables.

18. Au sens étymologique du terme.

19. Barlaam et Josaphat provençal, p.1157. Nous citons le texte dans la traduction de R. Nelli.

20. Ibid., p. 1193.

21. Mystère, v. 12026 sq.

22. Barlaam provençal, p. 1087.

23. Ibid., p. 1091. R. Nelli prend l'expression « cœur spirituel» pour un indice du caractère «cathare» de ce Barlaam.

24. Mise en prose, \& LXV.

25. Barlaam provençal, p. 1121.

26. Mise en prose, \& XLV.

27. L'expression est davantage réservée par l'Église aux époux chrétiens.

28. Josaphat impose sa foi par l'exemple de sa vie parfaitement juste et par nul autre moyen ; il n'est pas dit que Barrachis, qu'il désigne pour son successeur après la mort d'Avennir, était chrétien.

29. Barlaam provençal, p. 1197.

30. Mise en prose, \& CXVIII.

31. Version Champenoise, p. 137.

32. Barlaam provençal, p.1203.

33. Ibid., p. 1215.

34. Ibid., p. 1161.

35. Comme on le sait, le roman de Barlaam et Josaphat n'est en fait qu'un remake de la vie du Bouddha.

\section{AUTEUR}

\section{MARIE-GENEVIÈVE GROSSEL}

Université de Valenciennes 